\title{
Differential effects of offspring condition-dependent signals on maternal care regulation in the European earwig
}

\author{
Flore Mas • Mathias Kölliker
}

Received: 13 January 2010 /Revised: 11 May 2010 /Accepted: 23 August 2010 /Published online: 16 September 2010

(C) Springer-Verlag 2010

\begin{abstract}
Parent-offspring conflict theory predicts the evolution of offspring solicitation signals that can influence the amount and/or the duration of parental investment. Short-term effects of offspring solicitation signals on parental food provisioning have been widely demonstrated, but persistent effects of offspring signals on the maintenance of parental care have been rarely studied. Also, the relation between the amount of care provided to the brood and how it is distributed among individual offspring within a brood is not well enough understood. Here, we investigated in the European earwig (Forficula auricularia) the effects of offspring condition-dependent chemical signals on the maintenance of maternal care among broods and the distribution of maternal food within broods. Mothers were isolated from their brood for 3 days and continuously exposed to chemical signals extracted from broods of experimentally manipulated nutritional state. After re-introducing mothers to their brood, a range of maternal behaviours were quantified. We found that earwig mothers groomed their offspring significantly more after exposure to chemical extract from high-food brood in comparison with mothers exposed to extract from low-food brood, which in turn displayed significantly more aggressive behaviour. Furthermore, we manipulated offspring individual nutritional condition within the brood to evaluate the effect of offspring state on the within-brood food
\end{abstract}

Communicated by J. Choe

F. Mas $(\bowtie) \cdot$ M. Kölliker

Zoological Institute, Evolutionary Biology, University of Basel, Vesalgasse 1,

4051 Basel, Switzerland

e-mail: floremas@hotmail.com

M. Kölliker

e-mail: Mathias.koelliker@unibas.ch distribution. Within broods, poorly fed individuals received significantly more food than well-fed individuals, probably due to scramble competition. These results show that earwig nymphs express multi-component conditiondependent signals and behaviours differentially affecting maternal care provisioned to the brood and the distribution of care within broods.

Keywords Parent-offspring conflict · Scramble competition · Parental care - Chemical communication . Begging $\cdot$ Forficula auricularia

\section{Introduction}

A substantial body of empirical research has demonstrated the role of offspring condition-dependent signals in regulating parental care (see for reviews, Kilner and Johnstone 1997; Royle et al. 2002; Wright and Leonard 2002). Most of these studies manipulated the short-term nutritional condition (hunger) of offspring and, hence, tested shortterm, potentially transient, effects of offspring signals on the amount of food provisioned by parents. Fewer studies, particularly on colouration and UV-reflectance in birds (Lyon et al. 1994; Price and Ydenberg 1995; Saino et al. 2000; Bize et al. 2006; Tanner and Richner 2008) and recently on a chemical signal in an insect (Mas et al. 2009), also showed that condition-dependent signals not always function as signals of short-term "need" but sometimes reflect a longer-term nutritional effect as an offspring signal of "quality" or reproductive value. Thus, offspring signals may contain a range of information influencing parental investment in terms of the amount and/or maintenance and duration of care. In some mammals, the use of olfactory cues produced by the offspring 
was shown to affect the maintenance of maternal care (reviewed in Lévy and Keller 2009). In an earlier paper, we proposed that in insects, offspring solicitation pheromone may evolve to influence maternal physiology and the maintenance of care in the offspring's best interest (Mas and Kölliker 2008). Few empirical studies in insects measured the effect of offspring on the maintenance or duration of care, and these focussed on offspring age (e.g. Caussanel 1970; Klemperer 1983; Kight 1997). But it has been rarely demonstrated that an offspring signal per se can influence the maintenance of parental care behaviours.

Although parental food provisioning seems at first controlled by parents, some models and empirical studies have shown that it can also be controlled by offspring which, through scramble competition, may exaggerate their signal to attract resources from their passively responding parents (Parker and Macnair 1979; Kacelnik et al. 1995; Cotton et al. 1999; Parker et al. 2002). Competition between interacting offspring in a brood may influence food distribution within the brood, but coordination of signalling among offspring may also act in a cooperative manner by increasing the overall amount of food provided to the brood by parents (Leonard and Horn 1998; Krebs 2001; Leonard and Horn 2001a; Mathevon and Charrier 2004; Bell 2007; Horn and Leonard 2008). Understanding how potentially different offspring condition-dependent signals affect both the maintenance of care and the distribution of resources within the brood can thus provide insight into conflict resolution.

In this study, we tested if offspring condition-dependent signals and behaviours differentially affect the maintenance of maternal care and the distribution of food within broods in the European earwig, Forficula auricularia. This species displays characteristic maternal behaviours, such as brood defence, grooming (see Fulton 1924; Lamb 1976b) and food provisioning by trophallaxis (Staerkle and Kölliker 2008) until nymphs disperse. Recently, Mas et al. (2009) demonstrated an offspring chemical signal of quality based on cuticular hydrocarbons. Exposure of mothers to chemical cues extracted from well-fed nymphs lead to higher food provisioning to the brood than exposure to extracts from poorly fed nymphs or just solvent. Here, we tested if these recently discovered brood chemical signals of quality can also affect the longer-term maintenance of maternal care behaviours. Females were isolated for 3 days from their nymphs (i.e. mimicking dispersal of nymphs) and continuously exposed to chemical extracts of either highfood brood (HFB), low-food brood (LFB) or just the solvent (control C). If brood chemical signals of quality contribute to maintaining care in their mothers, we predicted that despite separation from their brood maternal behaviours should be maintained more when females were continuously exposed to chemical extracts from HFB. In addition, we manipulated individual nutritional condition of offspring within a same brood to test whether food distribution was random or whether it differed between offspring of different nutritional condition. In case of nonrandom food distribution, we were particularly interested in the direction of the effect of individual condition on the within-brood food distribution.

\section{Material and methods}

\section{Husbandry}

The earwigs used in our experiments originated from a population located in Gommiswald (Switzerland) and were caught in summer 2008. Husbandry conditions and general handling up to the hatching of clutches followed the protocols described in detail by Kölliker (2007) and Mas et al. (2009). For logistic reasons, that is, to spread out egg-laying and hatching, we split the lab population in two sub-populations that were set up either in July or October under a short-day photoperiod regime to trigger egg-laying (photoperiod/temperature: $8: 16 \mathrm{~h} / 20: 15^{\circ} \mathrm{C}$ (day-night) and $50 \%$ relative humidity).

At hatching, broods were randomly assigned to be used either for chemical extraction or to be kept with their mothers in family groups to be used in the behavioural tests. All hatched broods were transferred in new petri dishes $(10 \times 2 \mathrm{~cm})$ with humid sand and a shelter and were kept for the entire experiment under a long-day photoperiod regime of (photoperiod/temperature $16: 8 \mathrm{~h} / 20: 15^{\circ} \mathrm{C}$ and $50 \%$ relative humidity).

\section{Broods for chemical extraction}

The broods assigned for chemical extraction were standardised to 40 nymphs on day 1 after hatching by mixing two or three broods together and setting them up without a mother. On the first and second day, all broods received ad libitum food in the form of pellets of pollen (Kölliker 2007; Mas et al. 2009). On days 3 and 4, broods were randomly assigned to a food treatment, either broods continued to receive ad libitum food resulting in (HFB) or broods were not provided with food for the two successive days resulting in LFB. On day 5, LFB and HFB were frozen at $-20^{\circ} \mathrm{C}$. The 40 nymphs from each biosource brood were together immersed in $800 \mu \mathrm{l}$ of n-heptane (Rotisolv ${ }^{\circledR} 99 \%$ pure; Carl-Roth AG, Reinach, Switzerland) for $5 \mathrm{~min}$. For later use for female exposure, $600 \mu \mathrm{l}$ from these crude cuticular extracts were transferred in new glass vials $(2 \mathrm{ml}$, Sigma-Aldrich, Buchs, Switzerland). To avoid contamination, all the used glassware was cleaned three times each with three 
different solvents (heptane, acetone, dichloromethane; Carl-Roth AG, Reinach, Switzerland).

\section{Broods for behavioural assays}

The broods assigned for behavioural assays were formed from only one family and broods were culled to 20 nymphs and kept with their mother. They were provided with food daily from day 1 till day 4 after hatching. On day 5, mothers and nymphs were separated from each other and females were allocated randomly to the chemical exposure treatments. See Fig. 1 for an illustration of the experimental design.

Mothers exposure treatment On day 5, mothers were set up in a new petri dish $(10 \times 2 \mathrm{~cm})$ which included an exposure chamber. This exposure chamber consisted of a small petri dish $(3.5 \times 1 \mathrm{~cm})$ with openings cut on the side that could be opened or closed by simply turning the lid of the petri causing minimal disturbance. The exposure chamber was lined with a disc of filter paper $(3.5 \mathrm{~cm}$ diameter). From days 5 to 7 , mothers were randomly assigned to an exposure treatment: they were either exposed to HFB extract, LFB extract or the solvent n-heptane (control C). Each mother was daily exposed to $100 \mu \mathrm{l}$ of extract/solvent (equivalent of five nymphs). The extract/solvent was applied on the filter paper and allowed to dry for $30 \mathrm{~min}$ before the female was introduced to the exposure chamber. To ensure exposure, mothers were enclosed inside the exposure chamber for $3 \mathrm{~h}$ after which they were allowed to move in and out of the exposure chamber during their nocturnal active period. The likelihood that mothers were

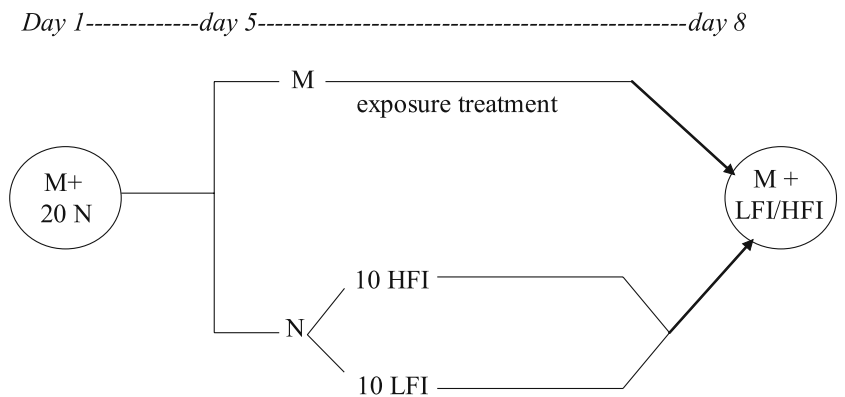

Fig. 1 Schematic figure of experimental setup. From days 1 to 4, mothers $(M)$ and 20 nymphs $(N)$ are kept together. On day 5, mothers are separated from their brood and randomly assigned to an exposure treatment: either extract from high-food brood $(H F B)$ or extract from low-food brood $(L F B)$ or pure heptan solvent as control $(C)$. Also on day 5 , the 20 nymphs are split in half and assigned to a condition treatment: ten nymphs on high-food treatment $(H F I)$ and ten nymphs on low-food treatment $(L F I)$. On day 8, mothers and their original brood, consisting of HFI and LFI, are gathered again. Maternal care behaviours were quantified during $1 \mathrm{~h}$ observation and mothers with their brood were let overnight together found inside or outside the exposure chamber on each day before renewing the extract was independent of the exposure treatment (GLM binomial (in/out): exposure treatments $F_{2,88}=0.07, p=0.92$, day $F_{2,169}=1.32 p=0.27$; exposure treatments $\times$ day, $\left.F_{4,169}=0.49, p=0.74\right)$ confirming that none of the solvent/extracts were overly repulsive or attractive. During these three consecutive days of exposure, mothers had no access to food in order to ensure that mothers would not accumulate extra food which they could not provide to their offspring. On day 8 , after $3 \mathrm{~h}$ of exposure, mothers were provided for $1 \mathrm{~h}$ with pellets of yellow pollen dyed with blue food dye as a marker (patent blue food dye; Werner Schweizer AG, Richterswil $\mathrm{CH}$ ) resulting in green coloration (see Staerkle and Kölliker 2008).

Offspring condition treatment During the 3 days of separation from their mothers, each brood was split in half and kept in separate petri dishes $(3.5 \times 1 \mathrm{~cm})$ resulting in split-brood of an average of $9.42 \pm 0.08$ nymphs. One half of the brood received ad libitum food in the form of pellets of pollen during the first 2 days (high-food individuals, HFI) whereas the other half did not receive any food (lowfood individuals, LFI). Because these split-broods would be gathered again later during the experiments, we randomly marked either all the HFI or LFI on their abdomen with a red marker pen (Potaco A.quip) in order to differentiate them. On day 7, both groups were deprived of food in order to standardise their short-term condition (i.e. variation in gut content) and increase their motivation for maternal food on day 8 when the behavioural experiment took place. The LFI and HFI groups from each family were gathered again together in a new petri dish $(10 \times 2 \mathrm{~cm})$ on the morning of the behavioural assays with the mother.

\section{Behavioural assays on day 8}

Assay 1: Maintenance of maternal behaviours In order to measure the effect of continuous exposure to brood chemical signals or the control solvent on the maintenance of maternal behaviour, we rejoined mothers with their original brood 3 days after separation and let them interact over $1 \mathrm{~h}$. During this hour, we used a scan sampling observational method (one scan observation every $5 \mathrm{~min}$; i. e. 12 scans per replicate) to record and quantify behaviours. We considered the following behavioural categories as maternal behaviours directed to offspring: (1) antennal contact with the nymphs, (2) mouth-to-mouth contact (this could either describe a mother trying to regurgitate food to a nymph or a nymph trying to trigger maternal regurgitation) and (3) grooming (female manipulating a nymph's body with her mouth parts). To obtain a measure of 
offspring rejection by mothers, we further quantified maternal aggression towards nymphs. We considered as aggressive behaviour when females were lifting their cerci upward in direction of a nymph, displaying the typical threat display of earwigs (Fulton 1924; Eisner 1960) or when shaking their abdomen while moving all over the petri dish to get rid of a nymph sitting on them (F.M., personal observation). Other female behaviours like scrubbing ventrally on the surface of the petri dish or digging close to the nymphs and carrying sand away could have been considered as maternal protection behaviours but since they were not obviously directed to or performed for the nymphs, we did not include them in the category of maternal behaviour. Finally, the most frequent behaviours such as exploring, self-grooming or resting were considered to be not specifically related to maternal care and although recorded, they were excluded from our statistical analysis. As a measure of mother-nymph aggregation, we scored at each scan if the closest group of two or more nymphs were within one female body length or not. Throughout these behavioural observations, the observer was blind with regard to the exposure treatments the mothers had been exposed to. Note that irrespective of the exposure treatment, the females always interacted with a brood consisting of approximately ten HFI and ten LFI nymphs and that the behavioural scores (grooming, antennal contact, mouth-to-mouth contact and aggression) in the scan samplings were not differentiated if they occurred between the mother and the HFI or the LFI nymphs.

Assay 2: Food distribution within brood After this observational hour, mothers were separated from the nymphs again for $1 \mathrm{~h}$ and allowed to forage on a second pellet of blue-dyed pollen. This step was considered to mimic a foraging trip. In order to assess the quantity of food eaten by mothers, pellets of food were weighed before and after each foraging trip with a Mettler Toledo AT261 balance with an accuracy of $0.001 \mathrm{mg}$. To control for effects of absorbed ambient humidity, the pellets were dried in the oven at $70^{\circ} \mathrm{C}$ prior to weighing. Finally, mothers were transferred back with their nymphs and allowed to interact overnight. The next morning (approximately $15 \mathrm{~h}$ later), provisioning to the brood was assessed by counting the total number of nymphs with green gut content and discriminating between LFI and HFI for food distribution within the brood (recognisable based on the marking; see above). Nymphs are fairly transparent and nymphs with food intake could be identified by their green gut content. Because only mothers had access to the green food, the proportion of nymphs with green gut content could be used as a measure of maternal provisioning (see Mas et al. 2009).
Statistical analysis

A total of 95 females with their brood were used in the behavioural assays. Four families were excluded from the statistical analysis because of female mortality before the end of the experiments resulting in a sample size of 91 . Females in the different exposure treatments did not differ in body mass (one-way ANOVA: $F_{2,89}=1.02$, $p=0.36$ ), clutch size (one-way ANOVA: $F_{2,89}=0.88$, $p=0.41$ ) or brood size at hatching (non-parametric Kruskal-Wallis: $H=4.38, p=0.11$ ) confirming proper randomization of treatment groups. The scores of maternal behaviours obtained from the scan observations were summed up for each mother to obtain frequencies of each behaviour over $60 \mathrm{~min}$. Given that most maternal behaviours in these scans showed skewed distributions and were rarely observed on average in the scans, we used generalised linear models (GLM) with a quasi-Poisson error distribution and a log-link function to test for differences in these maternal behaviours between the exposure treatments (extract from HFB or LFB or the solvent $\mathrm{C}$ ). In order to take into account potential trends over time during the observation period, we further performed an analysis where behaviours were summed over four successive periods of 15 min each. We then used a generalised linear mixed model (GLMM) with a quasiPoisson error distribution and a log-link function, with the frequencies of the behaviour as the dependent variable, time $(0-15,15-30,30-45$ and $45-60 \mathrm{~min})$ as a covariate, female identity as random factor and type of behaviours, exposure treatment and the interaction of exposure treatment with time as fixed factors.

To test the distribution of food within broods, we conducted a GLMM with a quasi-binomial error distribution and a logit function, number of nymphs with versus without green gut as odds ratio for the dependent variable, female identity as random factor, total food consumed by mother as covariate and nymph condition (HFI, LFI) as fixed factor. We used the statistical software R version 2.7.0 (R Development Core Team 2008).

\section{Results}

Effect of brood chemical signals on maintenance of maternal care behaviours

Earwig mothers spent most of their time performing nonmaternal activities such as exploring, self-grooming or resting (mean \pm S.E., $70 \pm 2 \%$ of total time). Behaviours considered as maternal or aggressive occurred in $28 \pm 2 \%$ and $2 \pm 0.7 \%$ of scan observations, respectively. Among these, there was a significant effect of the exposure treatment on maternal 
grooming of nymphs (GLM: $\chi^{2}=0.79, \mathrm{df}=89, p=0.036$ ) and aggressive behaviours against their nymphs (GLM: $\chi^{2}=1.72$, $\mathrm{df}=89, p=0.003$; Fig. 2). As predicted, mothers that were exposed to extracts from HFB groomed their nymphs significantly more often than mothers exposed to the solvent C (contrast: $t=1.94, p=0.030$ ) and more, though not significantly, than mothers exposed to extract from LFB (contrast: $t=2.20, p=0.055)$. Mothers exposed to extracts from LFB displayed significantly more often aggressive behaviours toward their nymphs compared to mothers exposed to extracts from HFB (contrast: $t=2.31, p=0.023$ ) or to solvent (contrast: $t=2.31, p=0.023$ ), for both of which almost no aggression was observed. The exposure treatment had no significant effect on the other maternal behaviours such as antennal contact (GLM: $\chi^{2}=10.29$, $\mathrm{df}=89, p=0.32$ ) and mouth-to-mouth contact (GLM: $\left.\chi^{2}=13.19, \mathrm{df}=89, p=0.25\right)$.

Mothers spent most of their time in the vicinity of their offspring irrespective of the exposure treatments (frequency of observations where at least two nymphs were within less than one female body distance $=95 \%, H=0.14, p=0.93$ ). When analysing the temporal dynamics of expression of behaviours over $60 \mathrm{~min}$, maternal behaviours showed no clear trends over time (GLMM: behaviours $\times$ time $F_{3,1343}=2.07$, $p=0.10$ ), and there was no significant interaction between time and exposure treatments with regard to either the summed frequency of all the maternal behaviours (GLMM: behaviours $\times$ time $\times$ exposure treatments $F_{6,1343}=1.35$, $p=0.23$ ) or the frequency of any specific maternal behaviour (all $p$ values $>0.3$ ).

Effect of offspring condition on within-brood food distribution

Only 32 out of 91 tested broods had nymphs with green gut content. The incidence of occurred provisioning did not differ significantly among the three exposure treatments
(GLM: $\chi^{2}=67.95, \mathrm{df}=88, p=0.99$ ). Among the broods where provisioning occurred, the proportion of nymphs with food intake was not significantly affected by the exposure treatment $\left(F_{2,28}=0.26, p=0.77\right)$ nor by an interaction between treatment and individual nymph condition $\left(F_{2,28}=1.18, p=0.32\right)$. For further analysis of within-brood food distribution among HFI and LFI nymphs the broods from the three exposure treatments were pooled.

The proportion of nymphs that received maternal food in a brood was significantly and positively correlated with the amount of food foraged by the mother $\left(F_{1,30}=11.22\right.$, $p=0.002$ ). Analysing the effect of nymph nutritional condition on within-brood food distribution revealed that significantly more nymphs from the low condition (LFI) had green gut contents as compared to nymphs from the high condition (HFI; $F_{1,31}=10.86, p=0.003$ ).

\section{Discussion}

Maternal behaviours and offspring signals affecting them co-evolve as interacting phenotypes (Kölliker et al. 2005; Smiseth et al. 2008), partly driven by selection from a conflict of interest between mothers and offspring over parental investment (Trivers 1974). Selection on parentoffspring interactions can arise due to effects of offspring signals on the amount and/or the duration of maternal care (Trivers 1974). We previously showed an effect of shortterm $(1 \mathrm{~h})$ exposure of females to condition-dependent brood chemical signals (cuticular hydrocarbons) on the amount of maternal food provisioning to nymphs (Mas et al. 2009). The present study demonstrates an additional function for condition-dependent brood chemical signals in maintaining specific maternal behaviours such as grooming and in affecting aggression towards offspring. The direction of effects are consistent between the two studies, with more care performed by females exposed to extracts from $\mathrm{HF}$
Fig. 2 Frequencies for behaviours considered maternal (antennal contact, mouth-tomouth contact and grooming) or aggressive expressed over 60 min after 3 days of separation and exposure treatment (extract from HFB or LFB or a solvent control C). Means and standard errors were obtained by backtransforming the estimates from the Poisson regression model to the linear scale

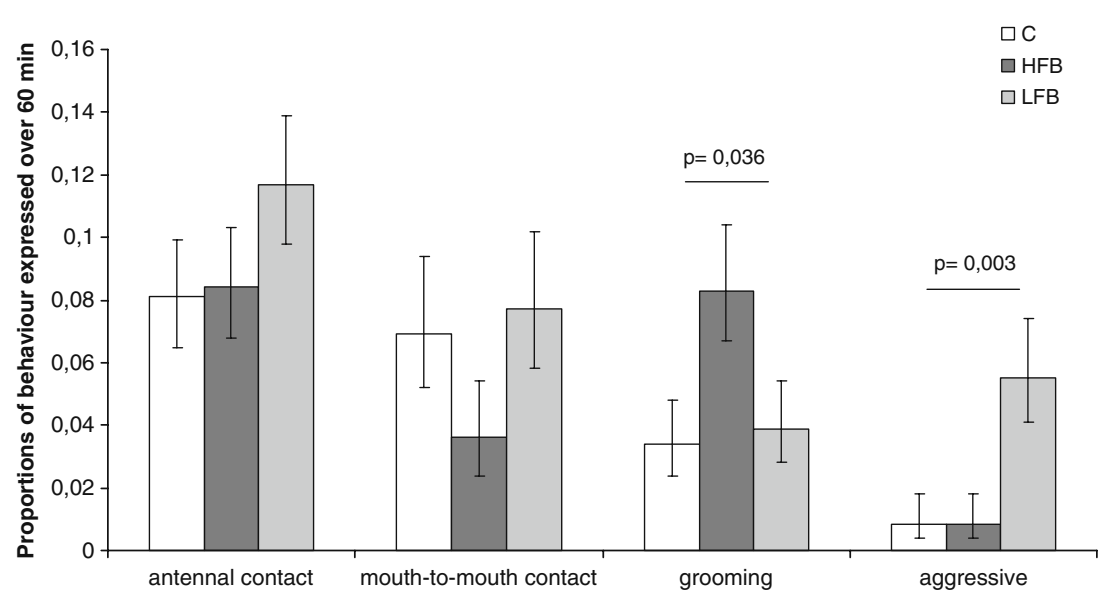


broods than LF broods, albeit with regard to different categories of maternal behaviour (food provisioning; Mas et al. 2009 and grooming/reduced aggression; this study).

Brood chemical signals and maintenance of maternal care

Although the function of grooming in maternal care is not immediately obvious, grooming clearly suggests that females tolerate physical proximity of nymphs, while aggression is indicative for the opposite effect. In earwigs, females are tolerant to their offspring during the maternal period but also display aggressive behaviours toward nymphs in particular after their dispersal (in F. auricularia, Lamb 1976b; and Labidura riparia, Vancassel 1977; Vancassel et al. 1987; Radl and Linsenmair 1991). Thus, the enhanced grooming by females exposed to HF extracts and the higher aggression by females exposed to LF extracts combined suggest a differential effect of condition-dependent chemical signals on the maintenance of care. By staying tolerant to their offspring, offspring can stay in proximity to their mothers and potentially gain protection benefit against predator attacks for longer, even at an age when food provisioning becomes less critical. Conversely, by behaving more aggressively toward nymphs, females exposed to cues indicating poor offspring quality may promote early dispersal of offspring and thus save maternal investment for her second brood. It remains to be tested in future studies whether the same chemical compounds from the total cuticular extract are responsible for the effects on provisioning and the other maternal behaviours.

The other behaviours that we considered as maternal like antennal contact and mouth-to-mouth contact did not differ significantly between the exposure treatments. Some of these results may at least partly be due to low statistical power, and our results on the maintenance of maternal behaviour may generally be rather conservative. The method of scan sampling used to screen several families at the same time may have underestimated the frequency of these types of maternal behaviours because they were relatively rare events and lasted for short time compared to behaviours like resting or cleaning (F.M., personal observation).

The previous study on earwig food provisioning (Mas et al. 2009) was based on short-term exposure of earwig mothers to extracts, that is, the immediate response in terms of food provisioning after exposure to condition-dependent offspring signals for $1 \mathrm{~h}$ (Mas et al. 2009). In the present study, it is conceivable that the low frequency and amount of provisioned food, and the lack of significant effect of exposure treatment, may in part be explained by the 3 days of separation of mothers from their brood, and the older age at which provisioning was quantified (day 8: this study; day 6: Mas et al. 2009). Food provisioning in earwigs is highest on days 3 and 4 after hatching and decreases afterwards (Kölliker 2007). Because with age earwig offspring start to leave the nest with their mothers for foraging (Lamb 1976a, b; F.M. and M.K., personal observation), maternal protection against predators may become relatively more important than maternal food provisioning.

Maternal care in F. auricularia has been reported to last until nymphs disperse (Lamb 1976b; Lamb 1976a). Vancassel and Foraste (1980a) showed that the presence of young nymphs maintains earwig maternal care behaviour, which can be empirically extended by preventing the nymphs from dispersing (Vancassel and Foraste 1980b). We demonstrated that the presence of condition-dependent brood chemical signals is sufficient to exert a persisting influence on some maternal behaviours, which might be correlated with the duration of care. Similar olfactory effects on maternal behaviour have been demonstrated in mammals (reviewed in Lévy and Keller 2009). For instance in sheep, females remain maternal after mother-young separation when olfactory cues are available (Poindron and Le Neindre 1980) and washing the lamb prevents acceptance behaviour while aggressive behaviour increases (Lévy and Poindron 1987). In general, in mammals, it is well understood how offspring signals act on the regulation of neuroendocrine factors and/or hormones mediating the expression of maternal behaviours (see Bridges 2008). These mechanisms are less studied in insects but a certain number of empirical studies have shown the effect of, yet unidentified, stimuli from offspring that regulate juvenile hormone titers of parents (i.e., in the ring-legged earwig, Euborellia annulipes Rankin et al. 1997; in burying beetle, Nicrophorus spp. Panaitof and Scott 2004; Scott and Panaitof 2004; Trumbo and Robinson 2008). Juvenile hormone is known to regulate development and reproduction in insects (Nijhout 1994) and is of particular importance in the regulation of parental care. In our study, we showed that chemical signals from offspring earwig maintained some maternal behaviours like grooming, which could potentially happen through an effect on the mother's physiological state, but this remains to be confirmed. If offspring chemical signals per se can influence the expression and maintenance of maternal behaviours such as grooming or aggression, offspring may potentially extend the duration of care provided by a mother in their best interest and at a cost for mother's future reproduction.

Offspring condition and within-brood food distribution

Independent from the exposure to the brood chemical signals, significantly more LFI than HFI nymphs received maternally provisioned food. Our experiment does not allow us to directly discriminate between an active choice from mothers to allocate food preferentially to LFI and the outcome of enhanced efforts of LFI nymphs to outcompete 
HFI nymphs in a scramble for maternally provisioned food. In the former case, our results would be consistent with a maternally selected honest signal of need. However, it would appear contradictory if earwig mothers selected for a signal of quality at the level of the brood (food provisioning, Mas et al. 2009; grooming/low aggression; this study), and for a signal of need at the level of the individual offspring within a brood. It is more likely that scramble competition between sibs drives the dynamics of food distribution within earwig broods, where competition can be harsh (as indicated by frequent siblicide and cannibalism, Dobler and Kölliker 2010).

Competitive asymmetries between offspring resulting from age or size differences, for instance, are expected to regulate scramble competition and several experimental studies on a variety of species showed that older and bigger offspring, presumably in better condition, received more maternal food than the other sibs (in birds, Price and Ydenberg 1995; Cotton et al. 1999; in burying beetles, Smiseth et al. 2006; Smiseth et al. 2007; Smiseth and Moore 2008). However, if competitive asymmetries are low and variation in nutritional need is large, a unit of food for offspring in poor condition (LFI) will be more beneficial than for high condition (HFI) offspring. LFI offspring are expected to have a higher payoff for competing more intensely (Parker et al. 2002), which in turn could promote higher competitive efforts and/or solicitation by LFI nymphs (Parker et al. 2002; Royle et al. 2002). Smiseth and Moore $(2007,2008)$ showed that in asynchronous broods of burying beetles, food-deprived or junior larvae spent more time begging than satiated or senior larvae. In our experiment, nymphs were all aged the same and the benefit of receiving food was probably higher for LFI than HFI despite the fact that the 2 days of food treatments might have had an effect on nymphs' growth and resulted in size differences. Thus, the most likely explanation for our result that food distribution was biassed towards LFI nymphs is through an effect of condition scramble competition. Conditiondependent non-signalling components of offspring behaviour (Lotem 1998) like positioning towards the mother or activity (personal observation) may lead to dynamics of food distribution within broods that can differ from the maternally selected brood signal.

Maternal care regulation and sibling interactions: a hypothetical scenario

Our results on maternal behaviours and food distribution raise the possibility of frequency-dependent effects of condition-dependent offspring behaviours on a signal perceived by the female at the level of the brood in $F$. auricularia. In a brood where there are a majority of nymphs in good condition (HFI), the overall chemical signals emanating from the entire brood would provide a signal of high brood quality (HFB) to mothers promoting maternal care. Conversely, in a brood where a majority of nymphs is in poor condition (LFI), mothers would perceive an overall brood chemical signal of low quality (LFB), which could result in reduction of maternal care to the brood, including the few HFI nymphs present in the brood. With such a maternal response at the level of the brood, the interest of HFI may be less divergent with the interest of LFI because of a mutual benefit of maintaining mothers to care. Thus, when mothers respond to offspring signals of quality (i.e. reproductive value) at the level of the brood, but allow offspring to scramble over food distribution within broods, selection may favour more cooperation among siblings because, under diminishing returns of food intake on condition, there is an added benefit of having siblings in better condition. Studies reporting interactions similar to the ones reported here were on acoustic offspring signals in birds (tree swallow, Tachycineta bicolor Leonard and Horn 2001b; and black-headed gulls, Larus ridibundus Mathevon and Charrier 2004; Horn and Leonard 2008), and banded mongoose, Mungos mungo (Bell 2007). In these species, individual offspring in a brood/litter not only beg at higher intensity when hungry and when competing with siblings, but they also adjust the calls to each other, resulting in a collective signal enhancing overall food provisioning to the entire brood. Another example of a brood signal was reported in the treehopper Umbonia crassicornis, where the offspring synchronise vibrational signals from all offspring, even the ones further from the predator, which in turn facilitates mothers to locate predators and to defend their brood (Cocroft 1996; Ramaswamy and Cocroft 2009). These arguments and examples indicate that offspring solicitation signals may sometimes combine competitive and cooperative components depending on how competitive interactions within broods affect brood signals that the caregiver uses to allocate investment between broods (Parker et al. 2002; Wilson and Clark 2002; Johnstone 2004; Forbes 2007).

In summary, our study on the European earwig demonstrates that condition-dependent brood chemical signals can have persistent effect on the maintenance of maternal behaviours over time. Consistent with previous results on maternal food provisioning to the brood (Mas et al. 2009), an increased in maternal grooming after exposure to extracts from high-food brood supports the hypothesis that earwig mothers may select for a brood chemical signal of quality. Condition-dependent effects on the distribution of food within broods differed from the effects of the brood chemical signal on maternal behaviours, suggesting an important role of multi-component offspring signals and behaviours differentially affecting amount and duration of care. 
Acknowledgements We thank Ralph Dobler, Joel Meunier, Janine Wong, Nicole Kalberer and David Duneau for their constructive comments on the manuscript and Martin Ackermann for the discussion. This project was financed by the Swiss National Foundation (grant no. PP00A-119190 to M.K.) and the Emilia Guggenheim-Schnurr-Stiftung (fellowship to F.M.).

\section{References}

Bell MBV (2007) Cooperative begging in banded mongoose pups. Curr Biol 17:717-721

Bize P, Piault R, Moureau B, Heeb P (2006) A UV signal of offspring condition mediates context-dependent parental favouritism. Proc R Soc Lond 273:2063-2068

Bridges RS (2008) Neurobiology of the parental brain. Academic, Elsevier

Caussanel C (1970) Main eco-physiological requirements of Labidura riparia. Ann Soc Entomol Fr 6:589-612

Cocroft RB (1996) Insect vibrational defence signals. Nature 382:679-680

Cotton PA, Wright J, Kacelnik A (1999) Chick begging strategies in relation to brood hierarchies and hatching asynchrony. Am Nat 153:412-420

Dobler R, Kölliker M (2010) Kin selected siblicide and cannibalism in the European earwig. Behav Ecol 21:257-263

Eisner T (1960) The chemical and mechanical weapons of an earwig. Psyche 67:62-70

Forbes S (2007) Sibling symbiosis in nestling birds. Auk 124:1-10

Fulton BB (1924) Some habits of earwigs. Ann Entomol Soc Am 17:357-367

Horn AG, Leonard ML (2008) Acoustic interactions in broods of nestling birds (Tachycineta bicolor). J Comp Psych 122:298-304

Johnstone RA (2004) Begging and sibling competition: how should offspring respond to their rivals? Am Nat 163:388-406

Kacelnik A, Cotton PA, Stirling L, Wright J (1995) Food allocation among nestling starlings: sibling competition and the scope of parental choice. Proc R Soc Lond 259:259-263

Kight SL (1997) Factors influencing maternal behaviour in a burrower bug, Sehirus cinctus (Heteroptera: Cydnidae). Anim Behav 53:105-112

Kilner R, Johnstone RA (1997) Begging the question: are offspring solicitation behaviours signals of needs. Trends Ecol Evol 12:11-15

Klemperer HG (1983) Subsocial behavior in Oniticellus cinctus (Coleoptera, Scarabaeidae): effect of the brood on parental care and oviposition. Physiol Entomol 8:393-402

Kölliker M (2007) Benefits and costs of earwig (Forficula auricularia) family life. Behav Ecol Sociobiol 61:1489-1497

Kölliker M, Brodie EDI, Moore AJ (2005) The coadaptation of parental supply and offspring demand. Am Nat 166:506-516

Krebs EA (2001) Begging and food distribution in crimson rosella (Platycercus elegans) broods: why don't hungry chicks beg more? Behav Ecol Sociobiol 50:20-30

Lamb RJ (1976a) Dispersal by nesting earwigs, Forficula auricularia (Dermaptera-Forficulidae). Can Entomol 108:213-216

Lamb RJ (1976b) Parental behavior in Dermaptera with special reference to Forficula auricularia (Dermaptera-Forficulidae). Can Entomol 108:609-619

Leonard ML, Horn AG (1998) Need and nestmates affect begging in tree swallows. Behav Ecol Sociobiol 42:431-436

Leonard ML, Horn AG (2001a) Acoustic signalling of hunger and thermal state by nestling tree swallows. Anim Behav 61:87-93
Leonard ML, Horn AG (2001b) Begging calls and parental feeding decisions in tree swallows (Tachycineta bicolor). Behav Ecol Sociobiol 49:170-175

Lévy F, Poindron P (1987) The importance of amniotic fluids for the establishment of maternal behavior in experienced and nonexperienced ewes. Anim Behav 35:1188-1192

Lévy F, Keller M (2009) Olfactory mediation of maternal behavior in selected mammalian species. Behav Brain Res 200:336-345

Lotem A (1998) The overlooked signaling component of nonsignaling behavior. Behav Ecol 10:209-212

Lyon BE, Eadie JM, Hamilton LD (1994) Parental choice selects for ornamental plumage in American coot chicks. Nature 371:240-243

Mas F, Kölliker M (2008) Maternal care and offspring begging in social insects: chemical signalling, hormonal regulation and evolution. Anim Behav 76:1121

Mas F, Haynes KF, Kölliker M (2009) A chemical signal of offspring quality affects maternal care in a social insect. Proc R Soc Lond 276:2847-2853

Mathevon N, Charrier I (2004) Parent-offspring conflict and the coordination of siblings in gulls. Proc R Soc Lond 271(Suppl 4): S145-7

Nijhout HF (1994) Insect hormones. Princeton University Press, Princeton

Panaitof SC, Scott MP (2004) Juvenile hormone and parental responsiveness in single male burying beetles, Nicrophorus orbicollis. Integr Comp Biol 44:734-734

Parker GA, Macnair MR (1979) Models of parent-offspring conflict. 4. Suppression: evolutionary retaliation by the parent. Anim Behav 27:1210-1235

Parker GA, Royle NJ, Hartley IR (2002) Begging scrambles with unequal chicks: interactions between need and competitive ability. Ecol Lett 5:206-215

Poindron P, Le Neindre P (1980) Endocrine and sensory regulation of maternal behavior in the ewe. In: Rosenblatt JS, Hinde RA, Beer $\mathrm{C}$ (eds) Advances in the study of behavior, vol 11. Academic, New York, pp 75-119

Price K, Ydenberg R (1995) Begging and provisioning in broods of asynchronously-hatched yellow-headed blackbird nestlings. Behav Ecol Sociobiol 37:201-208

R Development Core Team (2008) R: A language and environment for statistical computing. In, 2.7.0 edn. R foundation for statistical computing. Vienna, Austria

Radl RC, Linsenmair KE (1991) Maternal behavior and nest recognition in the subsocial earwig Labidura riparia Pallas (Dermaptera, Labiduridae). Ethology 89:287-296

Ramaswamy K, Cocroft RB (2009) Collective signals in treehopper broods provide predator localization cues to the defending mother. Anim Behav 78:697-704

Rankin SM, Chambers J, Edwards JP (1997) Juvenile hormone in earwigs: roles in oogenesis, mating, and maternal behaviors. Arch Insect Biochem Physiol 35:427-442

Royle NJ, Hartley IR, Parker GA (2002) Begging for control: when are offspring solicitation behaviours honest? Trends Ecol Evol $17: 434-440$

Saino N, Ninni P, Incagli M, Calza S, Sacchi R, Moller AP (2000) Begging and parental care in relation to offspring need and condition in the barn swallow (Hirundo rustica). Am Nat 156:637-649

Scott MP, Panaitof SC (2004) Social stimuli affect juvenile hormone during breeding in biparental burying beetles (Silphidae: Nicrophorus). Horm Behav 45:159-167

Smiseth PT, Moore AJ (2007) Signalling of hunger by senior and junior larvae in asynchronous broods of a burying beetle. Anim Behav 74:699-705 
Smiseth PT, Moore AJ (2008) Parental distribution of resources in relation to larval hunger and size rank in the burying beetle Nicrophorus vespilloides. Ethology 114:789-796

Smiseth PT, Ward RJS, Moore AJ (2006) Asynchronous hatching in Nicrophorus vespilloides, an insect in which parents provide food for their offspring. Funct Ecol 20:151-156

Smiseth PT, Lennox L, Moore AJ (2007) Interaction between parental care and sibling competition: parents enhance offspring growth and exacerbate sibling competition. Evolution 61:2331-2339

Smiseth PT, Wright J, Kölliker M (2008) Parent-offspring conflict and co-adaptation: behavioural ecology meets quantitative genetics. Proc R Soc Lond 275:1823-1830

StaerkleM,KöllikerM(2008)Maternalfoodregurgitation tonymphsin earwigs(Forficula auricularia). Ethology 114:844-850

Tanner M, Richner H (2008) Ultraviolet reflectance of plumage for parent-offspring communication in the great tit (Parus major). Behav Ecol 19:369-373

Trivers RL (1974) Parent-offspring conflict. Am Zool 14:249-264
Trumbo ST, Robinson GE (2008) Social and nonsocial stimuli and juvenile hormone titer in a male burying beetle, Nicrophorus orbicollis. J Insect Physiol 54:630-635

Vancassel M (1977) Le développement du cycle parental de Labidura riparia. Biol Behav 2:51-64

Vancassel M, Foraste M (1980a) Importance of contacts between female and larvae in some Dermaptera. Biol Behav 5:269-280

Vancassel M, Foraste M (1980b) Parental relations in Dermaptera. Reprod Nutr Dev 20:759-770

Vancassel M, Foraste M, Quris R, Strambi A, Strambi C (1987) The parental response of females Labidura riparia to young and its control. Comp Endocrinol 6:169-173

Wilson DS, Clark AB (2002) Begging and cooperation: an exploratory flight. In: Wright J, Leonard ML (eds) The evolution of begging: competition, cooperation and communication. Kluwer, Dordrecht, pp 43-64

Wright J, Leonard ML (2002) The evolution of begging. Competition, cooperation \& communication. Kluwer Academic, Netherlands 